\title{
COMPARISON BETWEEN A LOW-VOLTAGE ELECTRON MICROSCOPE AND CONVENTIONAL TEM FOR NUMBER SIZE DISTRIBUTION OF PRIMARY PARTICLES OF NANOMATERIAL POWDERS AND COLLOIDS
}

\author{
${ }^{1}$ Claire DAZON, ${ }^{2}$ Benoît MAXIT, ${ }^{1}$ Olivier WITSCHGER \\ ${ }^{1}$ Laboratoire de Métrologie des Aérosols, Institut National de Recherche et de Sécurité (INRS), Vandœuvre, \\ France, EU, claire.dazon@inrs.fr, olivier.witschger@inrs.fr \\ ${ }^{2}$ Cordouan Technologies, Cité de la photonique, Pessac, France, EU, \\ benoit.maxit@cordouan-tech.com
}

https://doi.org/10.37904/nanocon.2019.8617

\begin{abstract}
Nanomaterial powders and colloids are already a large industry and are expected to continue to grow rapidly. In the context of risk assessment associated with nanomaterials, characterization of nanoparticle size and morphology is required. Until now, the best method giving direct access to these parameters has been electron microscopy (EM), in particular, transmission electron microscopy (TEM). Although this method is widely used, several issues are highlighted such as cost, maintenance, sample representativity and damage for sensitive materials. Low-voltage transmission electron microscopes (LVTEMs) could be an alternative approach to solve some of these issues. This paper presents a first comparison between a benchtop LVTEM and a conventional device to determine the number size distribution of the constitutent particles of two polydispersed industrial powders $\left(\mathrm{TiO}_{2}\right.$ and $\left.\mathrm{SiO}_{2}\right)$ with particle sizes close to $100 \mathrm{~nm}$ and two colloids referenced for their particle size (ERM FD 304 and NM $300 \mathrm{~K}$ ). The samples were prepared with an optimized deposition protocol involving glow discharging and Alcian blue solution pre-treatment on the EM grids. The benchtop LVTEM produced a rather good resolution and the relative differences obtained for the median diameters $D_{50}$ are generally within $\pm 15 \%$. On the basis of these results, benchtop LVTEM could be promoted for identifying nanomaterials within the framework of risk assessment strategy.
\end{abstract}

Keywords: Benchtop electron microscope, nanomaterials, nanomaterial classification, constituent particles, size distribution, powders, colloids

\section{INTRODUCTION}

Nanomaterial powders and colloids are already a large industry (Piccinno et al., 2012 [1]; Saldanha et al., 2017 [2]). Improvement of the synthesis processes and the new properties highlighted at the nanoscale lead to the creation of new and innovative products, which suggests that this industry is expected to continue to grow rapidly. With nanoparticles being used increasingly at work, potential exposure of workers to the inhalation of released particles is possible throughout the lifecycle of the powders and liquid suspensions (Bekker et al., 2015 [3]; Debia et al., 2016 [4]). Since adverse health effects of nanoparticles are a growing concern, it remains challenging to evaluate the risks associated with nanoparticles in order to propose preventative measures.

The first step in a risk assessment strategy is the identification of the "nano" nature of a material (ISO/TS 12901-1, 2012 [5]). The reference criterion proposed by the European Commission (EC) is based on the number size distribution of constituent particles (EC, 2011 [6]). A powder or colloid is considered as a nanomaterial if more than $50 \%$ of the constituent particles in the number size distribution has at least one dimension under $100 \mathrm{~nm}$. This recommendation does not specify a particular method to obtain the number size distribution but it highlights the necessity to implement the most relevant and available methods in this purpose. 
Until now, the best method for directly obtaining particle size and shape has been electron microscopy (EM), in particular transmission electron microscopy (TEM) (Babick et al., 2016 [7]; De Temmerman et al., 2013 [8]). Although this method is widely used, the main issues highlighted are the high cost of the devices, heavy maintenance, high operator qualification for manipulating the microscopes, and the damage caused by the electron beam to the most sensitive samples (biological or polymers). Moreover, the sample representativity can be discussed with TEM since one can expect to count nearly 1000 constituent particles. However, no open literature demonstrates this clearly. In the particular context of risk assessment, risk prevention professionals do not always have the necessary skills in EM methods or the funding to invest in expensive devices.
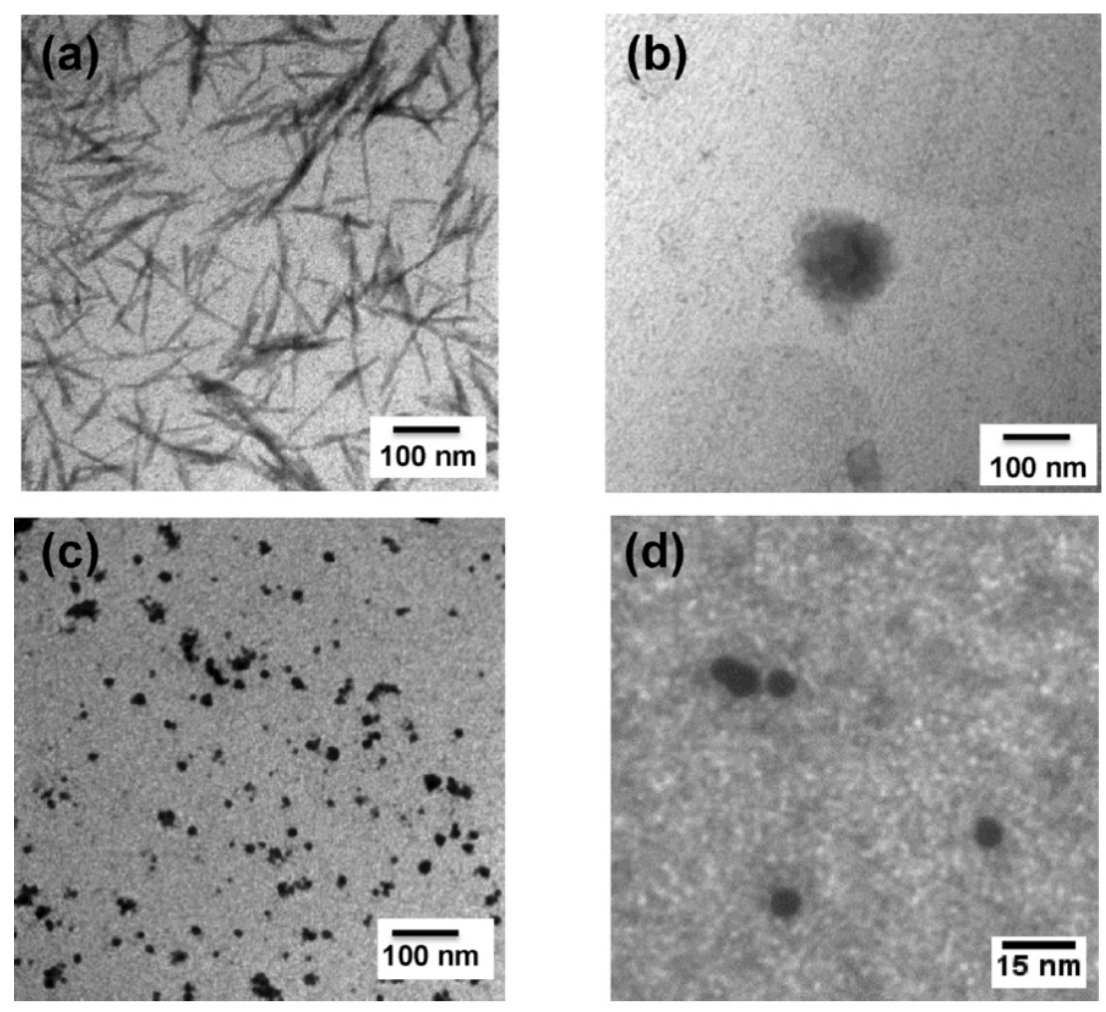

Figure 1 Examples of LVTEM applications for nanoparticle characterization. (a) cellulose fibers, (b) liposome, (c) nano-plastics collected in sea water and (d) goldnanoparticles

Recently, benchtop low-voltage transmission electron microscopes (LVTEMs) are evaluated as a solution to such issues (Delong, 1992 [9]; Bell et al., 2014 [10]; de la Calle et al., 2018 [11], Drummy, 2014 [12]). These unique instruments have been developed and manufactured by the company Delong Instrument (Brno, Czech Republic) since 1992 (Delong, 1992 [9]). Specifically dedicated to low voltage, these TEM are proposed in two versions: the LVEM5 operating at $5 \mathrm{kV}$ and the LVEM 25 at $25 \mathrm{kV}$. These instruments are extremely compact, require little space and resources and can combine other imaging modes such as scanning electron microscopy (SEM), scanning transmission electron microscopy (STEM) or electron diffraction (ED) in one single device. Moreover, low acceleration voltage combined with the Schottky field emission gun opens up the possibility of avoiding heavy metal stains typically used for imaging polymeric and organic molecular materials while causing less damage to this kind of material (Drummy, 2014 [12]) and preserving a reasonably high resolution $(<2 \mathrm{~nm}$ ). Figure 1 shows four examples of applications of LVTEM to characterize nanomaterials. Examples (a)-(c) respectively concern cellulose, liposome and nano-plastics collected in sea water. They present a low contrast when conventional TEM is used and can even be damaged by the electron beam at high voltage. With a $5 \mathrm{kV}$ low-voltage microscope, it becomes possible to characterize their shape and measure their diameters without using any staining. Example $(d)$ is a micrograph of gold nanoparticles between 
5 and $10 \mathrm{~nm}$ in diameter. This latter example shows that the resolution of LVTEM at $5 \mathrm{kV}$ allows nanoscaled particles to be characterized.

Therefore, LVTEM could offer some potential benefits for nanomaterial characterization with a risk assessment strategy, in particular, easier implementation and wider accessibility of the technique. However, no study to our knowledge has ever demonstrated the performance of LVTEM in determining number size distribution of powder or colloid nanoparticles with a view to implementing the first step of risk assessment strategy.

In this work, we evaluated the relevancy of a LVTEM by comparing the number size distributions of the constituent particles of two representative industrial powders $\left(\mathrm{TiO}_{2}\right.$ and $\left.\mathrm{SiO}_{2}\right)$ and two colloids referenced for their particle sizes based on the micrographs obtained with those from a conventional TEM device. The final goal was to have initial feedback about the possible use of LVTEM for the implementation of risk assessment strategy where nanomaterial identification is concerned.

\section{MATERIAL AND METHODS}

\subsection{Instrumentation}

Two different electron microscopes were used in this work. A Delong LVEM5 operating in transmission electron microscopy mode with a Schottky field emission gun at an accelerating voltage of $5 \mathrm{kV}$ and a spherical aberration coefficient $\mathrm{C}_{\mathrm{s}}$ of $0.64 \mathrm{~mm}$, and a conventional TEM CM200 (Philips) operating at an accelerating voltage of $200 \mathrm{kV}$ with a $\mathrm{C}_{\mathrm{s}}$ of $2 \mathrm{~mm}$ were implemented. The micrographs were collected with a Retiga 4000R CCD $2048 \times 2048$ for LVEM5 and a Gatan ES500W CCD $1350 \times 1024$ for CM200.

\subsection{Powders and colloids}

The characteristics of the materials are summarized in Table 1 based on manufacturers' information contained in commercial product data sheets. TiO2 is mainly used as colorant for paintings whereas $\mathrm{SiO} 2$ is employed in buildings for improving the mechanical properties of concretes. ERM FD 304 and NM $300 \mathrm{~K}$ are reference materials for their particle sizes. They are widely used in EM analysis as standard materials for checking devices (Thunemann et al., 2014 [13]). The same batch of each material was used to prepare the samples.

Table1 Characteristics of the powders and reference colloids used in this work according to data from the manufacturers

\begin{tabular}{|c|c|c|c|c|c|}
\hline Type & Material & $\begin{array}{c}\text { Specific Surface } \\
\text { Area }\left(\mathrm{m}^{2} / \mathrm{g}\right)\end{array}$ & $\begin{array}{l}\text { Particle } \\
\text { shape }\end{array}$ & $\begin{array}{l}\text { Particle size }(\mathrm{nm}) \\
\text { (reference values) }\end{array}$ & $\begin{array}{c}\text { Other } \\
\text { information }\end{array}$ \\
\hline \multirow[t]{2}{*}{ Powder } & TiO2 (anatase) & 9 & Spherical & N.A & $\begin{array}{l}\text { Industrial } \\
\text { powder }\end{array}$ \\
\hline & SiO2 (amorphous) & 23 & & & \\
\hline Colloid & ERM FD 304 (SiO2) & N.A & & $27.8 \pm 1.5^{\star}$ & $\begin{array}{c}\text { Material } \\
\text { certified for the } \\
\text { particle size } \\
\text { mode }\end{array}$ \\
\hline & NM300K (Ag) & & & $\begin{array}{l}17 \pm 3.17\left(1^{\text {st }} \text { population }\right) \\
5 \pm 1.4\left(2^{\text {nd }} \text { population }\right)\end{array}$ & $\begin{array}{l}\text { Reference } \\
\text { material for } \\
\text { mean size }\end{array}$ \\
\hline
\end{tabular}

\footnotetext{
* The uncertainties indicated for the reference materials correspond to one standard deviation available in literature.
} 


\subsection{Sample preparation and analysis}

For the powders, samples were prepared at the concentration $0.5 \mathrm{mg} / \mathrm{mL}$ in deionized water under basic conditions ( $\mathrm{pH} 10.5$ for $\mathrm{TiO}_{2}, \mathrm{pH} 11$ for $\mathrm{SiO}_{2}$ ) followed by $10 \mathrm{~min}$ of sonication at $50 \mathrm{~W}$ before grid deposition. These formulations correspond to the highest stabilities we could obtain for the dispersed particles in the medium based on previous zeta potential measurements. The reference colloids were only diluted by a factor 1000, then, sonicated $10 \mathrm{~min}$ at $50 \mathrm{~W}$. For LVEM5 observations, Holey carbon film Cu 300 Mesh grids (Cu$300 \mathrm{HD}$, Pacific Grid tech) were treated beforehand by glow discharge (ELMO, Cordouan Tech.) for $15 \mathrm{~s}$ at 2 $\mathrm{mA}$, followed by Alcian Blue $1 \%$ (Clin- Tech) treatment. Then, a $5 \mu \mathrm{L}$ droplet of colloid was deposited on the treated grids over the course of $1 \mathrm{~min}$ ("drop on grid method") before being blotted with a filter paper. The glow discharge plasma treatment makes the carbon membrane hydrophilic improving the wettability of the TEM grid for the colloidal drop, while the Alcian blue treatment charges the carbon surface positively, increasing its affinity with the negatively charged particles. The combination of both treatments improves the dispersion homogeneity of particles on the grid surface and limits the aggregation phenomenon. For TEM CM200 observations, Cu 400 Mesh carbon film grids (Agar Scientific) were deposited over the course of 1 min on 2 $\mu \mathrm{L}$ colloid drop ("grid on drop method") (Mast and Demeestere, 2009 [14]). We did not use the same type of grids in both microscopes since the grid membrane thickness has a high impact on the low voltage observation quality. Actually, the thinner the membrane is, the better resolution and contrast are, and it appeared that the best grids for the LVEM5 were constituted of a carbon membrane thinner than $5 \mathrm{~nm}$. On the other hand, these grids revealed to be too fragile for the TEM CM200. Therefore, we chose to use the most appropriate grids for each acceleration voltage in order to operate in the optimal conditions, and the best resolution, with each instrument.

To limit variability in the results, a single operator took and analyzed the micrographs obtained with LVEM5 and CM200 with the ImageJ software (Java version 1.8.0). A minimum of 100 isolated constituent particles were counted and their equivalent projected surface diameters were measured to establish the number size distributions since approximated spherical shape was observed for all the materials. To compare the results, the median diameters $D_{50}$ were calculated from the cumulated number size distributions.

\section{RESULTS AND DISCUSSION}

Figure 2 presents a typical micrograph obtained with LVEM5 and TEM CM200 for the four materials.

For all the materials, the particles are correctly dispersed on the grids. This facilitates the counting to determine the number size distribution. The ERM FD 304 sample presents the highest density of particles on the grids. Despite the high dilution factor (1000), the number of particles obtained on a single micrograph is much higher than for $\mathrm{TiO}_{2}, \mathrm{SiO}_{2}$ and NM $300 \mathrm{~K}$. LVEM5 shows a good resolution and a higher contrast compared to the conventional TEM CM200 for the material tested. It is possible to distinguish several constituent particles both with LVEM5 and TEM CM200. The particle shapes are nearly spherical for $\mathrm{SiO}_{2}$, ERM FD 304 and NM $300 \mathrm{~K}$ whereas $\mathrm{TiO}_{2}$ particles seem more ovoid.

Figure 3 represents the number size distributions and the corresponding cumulated number size distributions obtained on LVEM5 and TEM CM200 for the four materials. There is a large range of $\mathrm{TiO}_{2}$ and $\mathrm{SiO}_{2}$ particle sizes, from $30 \mathrm{~nm}$ to over $300 \mathrm{~nm}$; the ERM FD 304 and NM $300 \mathrm{~K}$ particle size range was smaller, from 18 $\mathrm{nm}$ to $36 \mathrm{~nm}$ and from $10 \mathrm{~nm}$ to $35 \mathrm{~nm}$ respectively. This illustrates the polydispersity of the industrial powders contrary to the reference colloids which are characterized by a monomodal number size distribution. There is a general good match between the number size distributions obtained from the two different sets of micrographs. However, larger differences are observed for the $\mathrm{SiO}_{2}$ particles, since the count obtained with the LVEM5 indicates a majority of constituent particles between 30 and $180 \mathrm{~nm}$ in size, while there is a wider size range, from $30 \mathrm{~nm}$ to $250 \mathrm{~nm}$, obtained with the TEM CM200. In our opinion, the sample preparation seems 
to be the main reason explaining such discrepancy since the same batch of powder was used. In particular, very few $\mathrm{SiO}_{2}$ particles larger than $200 \mathrm{~nm}$ were identified on the CM200 sample grid and not on the LVEM5 sample grid, despite of the significant number of micrographs observed for particles counting. Moreover, the change in contrast induced by the different acceleration voltages does not appear as a significant source of size disparity here since the $\mathrm{NM} 300 \mathrm{~K}, \mathrm{TiO}_{2}$ and $\mathrm{SiO}_{2}$ particles edges are quite sharp and dense.

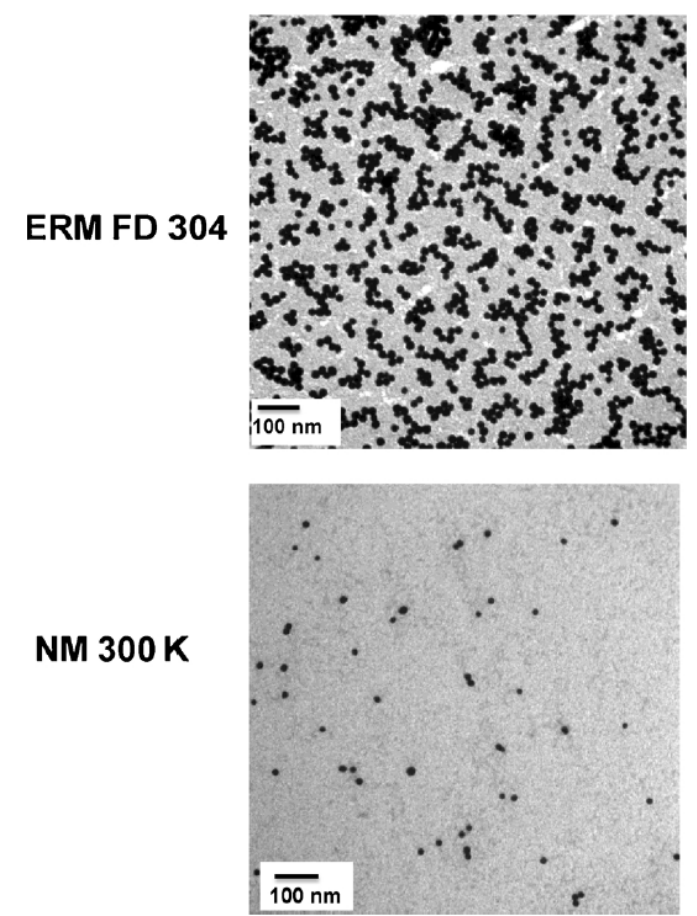

LVEM5
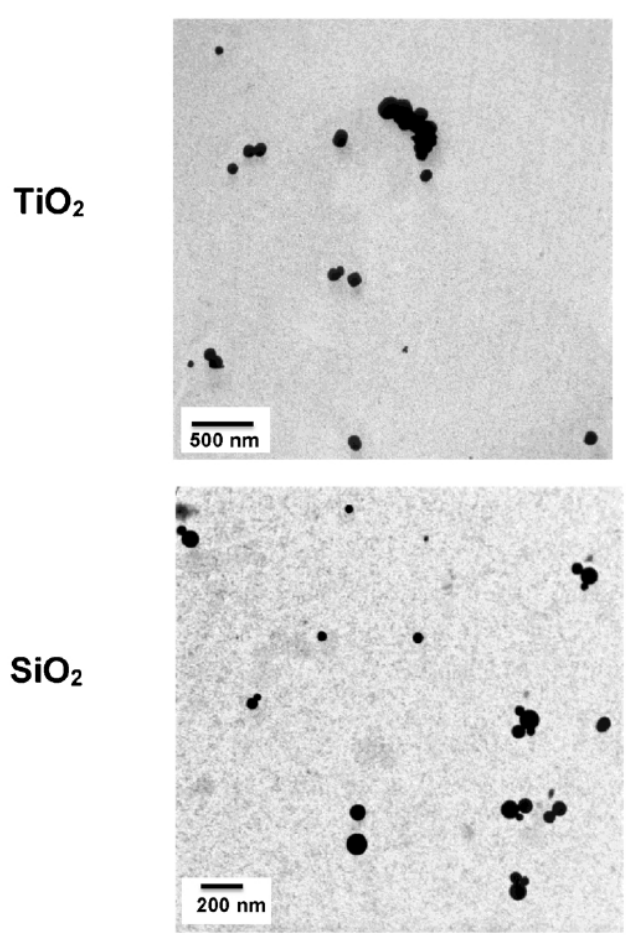
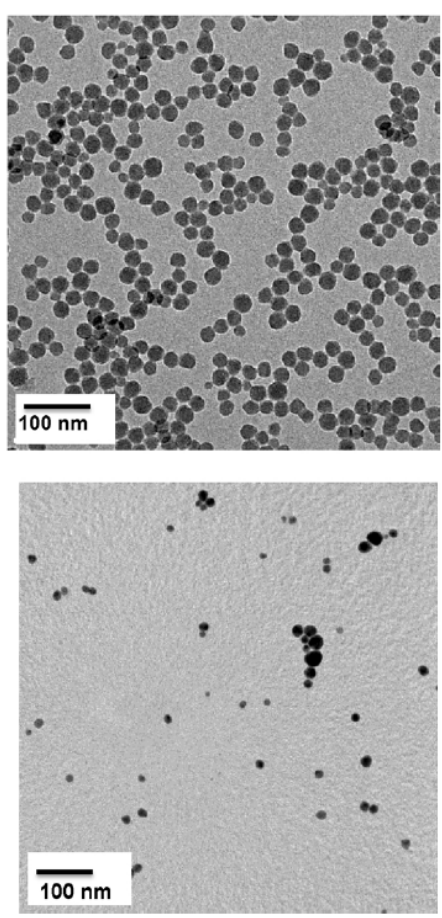

TEM CM200
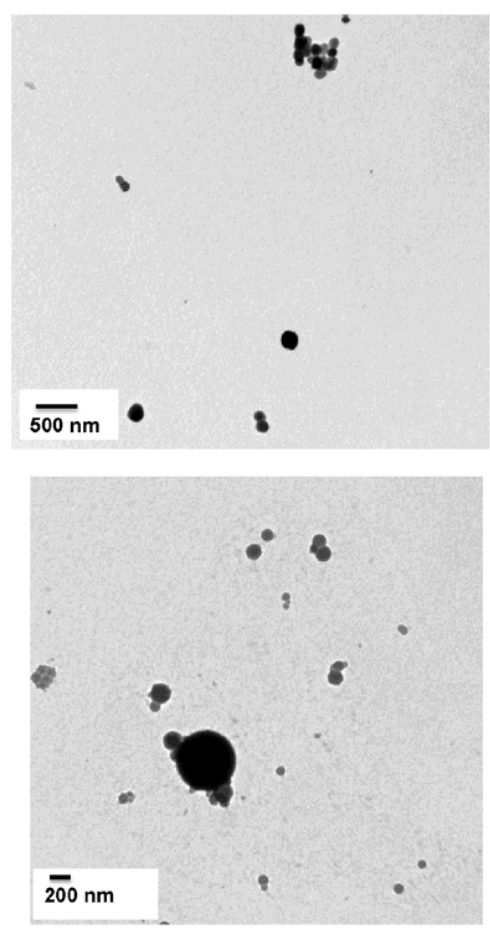

Figure 2 Typical micrographs obtained for the four materials studied with LVEM5 and TEM CM200 

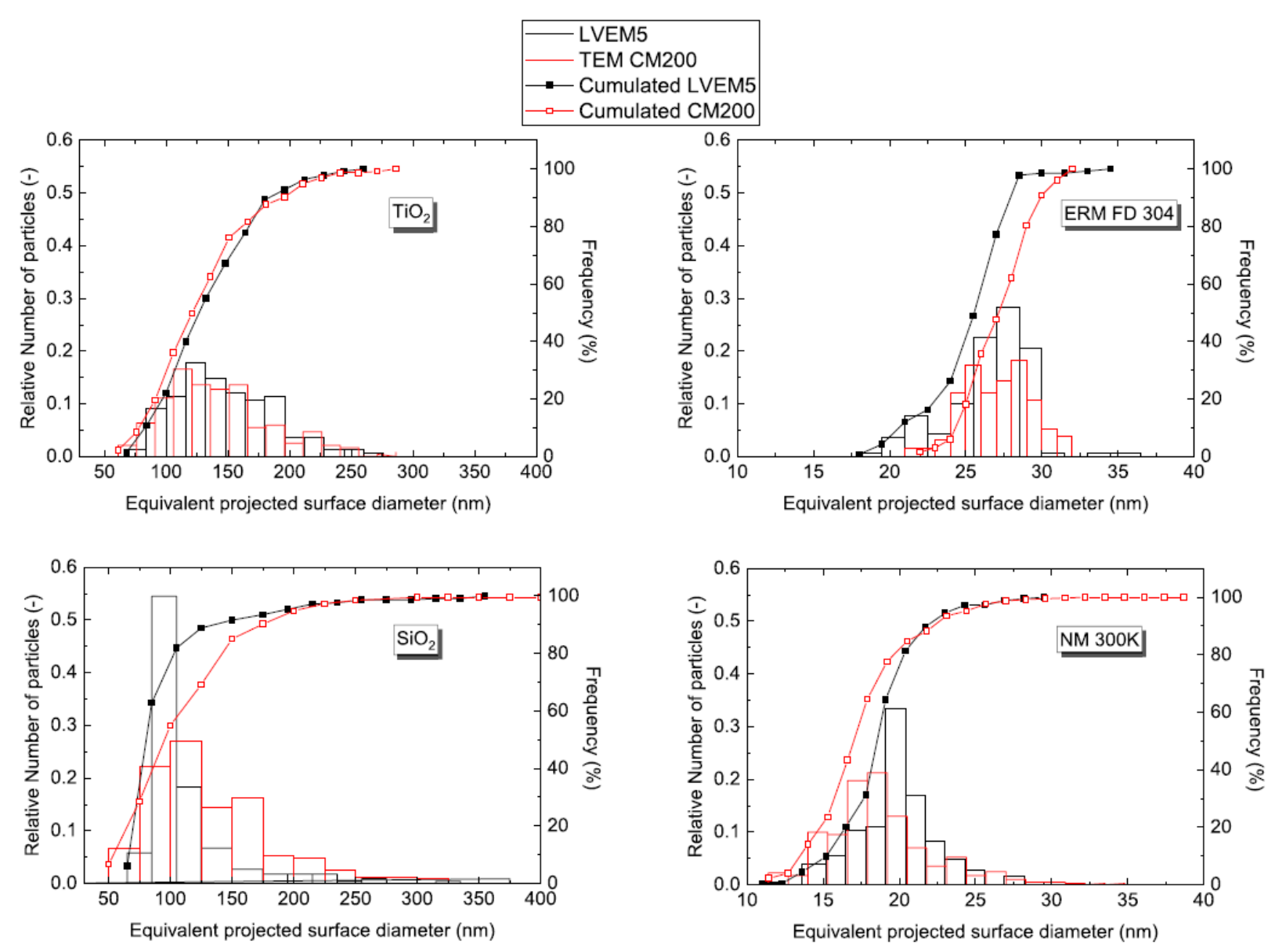

Figure 3 Number size distributions and cumulated number size distributions obtained with LVEM5 and TEM CM200 for the $\mathrm{TiO}_{2}$ and $\mathrm{SiO}_{2}$ powders and the ERM FD304 and NM $300 \mathrm{~K}$ colloids

Table 2 summarizes the $D_{50}$ obtained from the cumulated number size distributions. It can be seen that the LVEM5 results are in line with those of TEM CM200. In particular, the discrepancies of LVEM5 compared to TEM CM200 are within $\pm 15 \%$. The standard deviations are higher for the powders than for the colloids since the constituent particles are more agglomerated and more difficult to identify for counting. The best comparison is obtained for $\mathrm{TiO}_{2}$ with a relative difference of $2.5 \%$. The reference diameter is found for ERM FD 304 with both microscopes and the standard deviations associated are low. So, we validate the reference value obtained.

Table 2 Median diameters D50 obtained from the cumulated number-based size distributions. The values are indicated with one standard deviation

\begin{tabular}{cccccc}
\hline \multirow{2}{*}{ Material } & \multicolumn{2}{c}{ LVEM5 } & \multicolumn{2}{c}{ TEM CM200 } & Relative difference \\
\cline { 2 - 4 } & D50 $(\mathrm{nm})$ & $\%<100 \mathrm{~nm}$ & D50 $(\mathrm{nm})$ & $\%<100 \mathrm{~nm}$ & \\
\hline (LVEM5 vs.TEM CM200)
\end{tabular}


However, the ideal case is treated here. The ERM FD 304 is a monomodal spherical nanoparticulate sample and can be well characterized in terms of size with a quite reduced number of particles, typically around 100 particles. This result cannot be generalized, in particular for the powder cases where a greater number of particles should be counted (more than 1000 particles) to obtain as most as possible representativity as demonstrated by De Temmerman et al (De Temmerman et al., 2013 [8]). For NM $300 \mathrm{~K}$, the first reference diameter is found with both microscopes but the second diameter is not. The literature reports that the second diameter for this colloid is rarely observed (Klein et al., 2011 [15]). However, the main cause we attributed to this observation is a possible dissolution of the $5 \mathrm{~nm}$ population under acidic conditions as several studies highlighted this behavior for silver nanoparticles (Liu and Hurt, 2010 [16]; Loza et al., 2014 [17]; Zhang et al., 2018 [18]). Even if successive rinsing steps were done during grid preparation, some Alcian blue traces could have stayed on the grids and dissolve the finest silver nanoparticles. Indeed, Alcian blue solution contains $3 \%$ acetic acid. There is no general trend concerning the discrepancies and it can be supposed that the discrepancies observed in size measurement are mainly related to the sample preparation and the level of deagglomeration which would affect the operator's ability to identify isolated constituent particles. On the basis of the $\mathrm{D}_{50}$ results, both microscopes classify the $\mathrm{TiO}_{2}$ powder as a non-nanomaterial since more than $50 \%$ of its number size distribution of the constituent particles is higher than $100 \mathrm{~nm}$. The $\mathrm{SiO}_{2}$ powder and the reference colloids are classified as nanomaterials. We point out however that the two powders are border cases for both microscopes taking into account the influence of sample preparation and the relative high standard deviations obtained. Other characterization approach we performed apart from this study, such as the Volume Specific Surface Area (VSSA) determination (Wohlleben et al., 2017 [19]), highlighted these border line situations. A powder is considered as a nanomaterial for a VSSA value above $60 \mathrm{~m}^{2} / \mathrm{cm}^{3}$ according to the EC recommendation (EC, 2011 [6]). The VSSA values are of $35 \mathrm{~m}^{2} / \mathrm{cm}^{3}$ and $58 \mathrm{~m}^{2} / \mathrm{cm}^{3}$ for $\mathrm{TiO}_{2}$ and $\mathrm{SiO}_{2}$ respectively. Although the VSSA is not the reference criterion to classify a powder in the nanomaterial category, these results encourage to classify the $\mathrm{TiO}_{2}$ powder as a non-nanomaterial whereas the $\mathrm{SiO}_{2}$ case is confirmed to be very closed to the thresholds for size and VSSA parameters. In the risk assessment context, a borderline material should be classified as nanomaterial. So, $\mathrm{SiO}_{2}$ powder is proposed as a nanomaterial here.

\section{CONCLUSION}

In this work, we compared a low voltage benchtop electron microscope operating in transmission mode with a reference TEM device for subsequent establishment of number size distributions of nanoparticulate powders and colloids. Such work integrated a workplace exposure context to nanomaterials. The results demonstrated that benchtop LVTEM is a suitable device for generating quality micrographs with a resolution comparable to its TEM equivalent. The comparison of the number size distributions and the median diameters $D_{50}$ of the constituent particles obtained showed relative differences systematically within $\pm 15 \%$ which is relatively good taking into account the standard deviations obtained. For counting alone, a well-prepared sample and any one of the two microscopes would indicate whether or not industrial substances are nanomaterials and confirm the reliability of the EM method for size and shape determination with the use of references. Lastly, the comparison suggests that benchtop LVTEM could be used by risk prevention professionals in routine checks to identify nanomaterials more easily (facility of implementation) than with conventional TEM. However, these results need further investigation. It would be interesting to compare benchtop LVTEM and TEM on more industrial powders and colloids covering wider size ranges, different shapes (rod-like, fiber-like, platelets, needle-like, etc.) and chemical compositions. Moreover, the study of the influence of sample preparation and the repeatability of the measurements with benchtop LVEM should be undertaken to strengthen these first encouraging results. 


\section{ACKNOWLEDGEMENTS}

\section{The authors thank the Institut Jean Lamour and the Centre de Compétences en Microscopie Electronique et Microsonde for making the TEM CM200 available.}

\section{REFERENCES}

[1] PICCINNO, F., GOTTSCHALK, F., SEEGER, S., NOWACK, B., 2012. Industrial production quantities and uses of ten engineered nanomaterials in Europe and the world. J. Nanopart. Res.14, 1109.

[2] SALDANHA, P.L., LESNYAK, V., MANNA, L., 2017. Large scale syntheses of colloidal nanomaterials.Nano Today 12, 46-63.

[3] BEKKER, C., KUIJPERS, E., BROUWER, D.H., VERMEULEN, R., FRANSMAN, W., 2015. Occupational exposure to nano-objects and their agglomerates and aggregates across various life cycle stages: a Broad-scale exposure study. Ann. Occup. Hyg. 59, 681-704.

[4] DEBIA, M., BAKHIYI, B., OSTIGUY, C., VERBEEK, J.H., BROUWER, D.H., MURASHOV, V., 2016. A systematic review of reported exposure to engineered nanomaterials. Ann. Occup. Hyg. 60, 916-935.

[5] ISO, ISO/TS 12901-1:2012 (November 2012) Nanotechnologies - Occupational risk management applied to engineered nanomaterials - Part 1: Principles and approaches. Case postale 56. $\mathrm{CH}-1211$ Geneva 20, ISO copyright office, $37 \mathrm{p}$.

[6] EC, 2011. Commission recommendation of 18 October 2011 on the definition of nanomaterial. Offic. J. Eur. Union L275, 38-40.

[7] BABICK, F., MIELKE, J., WOHLLEBEN, W., WEIGEL, S., HODOROABA, V.-D., 2016. How reliably can a material be classified as a nanomaterial? Available particle-sizing techniques at work. J. Nanopart. Res. 18, 158.

[8] DE TEMMERMAN, P.-J., LAMMERTYN, J., DE KETELAERE, B., KESTENS, V., ROEBBEN, G., VERLEYSEN, E., MAST, J., 2013. Measurement uncertainties of size, shape, and surface measurements using transmission electron microscopy of near-monodisperse, nearspherical nanoparticles. J. Nanopart. Res. 16, 2177.

[9] DELONG, A., 1992. Electron Microscopy 1, 79-82.

[10] BELL, D.C., MANKIN, M., DAY, R.W., ERDMAN, N., 2014. Successful application of Low voltage electron microscopy to practical materials problems. Ultramicroscopy 145, 56-65.

[11] DE LA CALLE, I., MENTA, M., KLEIN, M., MAXIT, B., SEBY, F., 2018. Towards routine analysis of TiO2 (nano-)particle size in consumer products: evaluation of potential techniques. Spectrochim. Acta B: Atom. Spectroscopy 147, 28-42.

[12] DRUMMY, L.F., 2014. Electron microscopy of organic-inorganic interfaces: Advantages of low voltage. Ultramicroscopy 145, 74-79.

[13] THUNEMANN, A.F., EMMERLING, F., HODOROABA, V.-D., 2014. Review of existing calibration or reference materials. Nano Define Technical Report D1.1. NanoDefine Consortium, Wageningen.

[14] MAST, J., DEMEESTERE, L., 2009. Electron tomography of negatively stained complex viruses: application in their diagnosis. Diagn. Pathol. 4, 5.

[15] KLEIN, C., COMERO, S., STAHLMECKE, B., ROMAZANOV, J., KUHLBUSCH, T., VAN DOREN, E., et al., 2011. NM-Series of Representative Manufactured Nanomaterials: NM-300 Silver Characterisation, Stability, Homogeneity, EUR 24693 EN. Publications Office of the European Union, Luxembourg.

[16] LIU, J., HURT, R.H., 2010. Ion release kinetics and particle persistence in aqueous nanosilver colloids. Environ. Sci. Technol. 44, 2169-2175.

[17] LOZA, K., DIENDORF, J., SENGSTOCK, C., RUIZ-GONZALEZ, L., GONZALEZ-CALBET, J.M., VALLET- REGI, M., et al., 2014. The dissolution and biological effects of silver nanoparticles in biological media. J. Mater. Chem. B. 2, 1634-1643.

[18] ZHANG, W., XIAO, B., FANG, T., 2018. Chemical transformation of silver nanoparticles in aquatic environments: mechanism, morphology and toxicity. Chemosphere 191, 324-334.

[19] WOHLleBEN, W., MIELKE, J., BIANCHIN, A., GHANEM, A., FREIBERGER, H., RAUSCHER, H., et al., 2017. Reliable nanomaterial classification of powders using the volume-specific surface area method. J. Nanopart. Res. $19,61$. 